\title{
Planar Octilinear Drawings with One Bend Per Edge ${ }^{\star}$
}

\author{
Michael A. Bekos ${ }^{1}$, Martin Gronemann ${ }^{2}$, Michael Kaufmann ${ }^{1}$, and Robert Krug ${ }^{1}$ \\ 1 Wilhelm-Schickard-Institut für Informatik, Universität Tübingen, Germany \\ \{bekos, mk, krug\}@informatik. uni-tuebingen. de \\ 2 Institut für Informatik, Universität zu Köln, Germany \\ gronemann@informatik.uni-koeln.de
}

\begin{abstract}
In octilinear drawings of planar graphs, every edge is drawn as an alternating sequence of horizontal, vertical and diagonal line-segments. In this paper, we study octilinear drawings of low edge complexity, i.e., with few bends per edge. A $k$-planar graph is a planar graph in which each vertex has degree less or equal to $k$. In particular, we prove that every 4-planar graph admits a planar octilinear drawing with at most one bend per edge on an integer grid of size $O\left(n^{2}\right) \times O(n)$. For 5-planar graphs, we prove that one bend per edge still suffices in order to construct planar octilinear drawings, but in super-polynomial area. However, for 6-planar graphs we give a class of graphs whose planar octilinear drawings require at least two bends per edge.
\end{abstract}

\section{Motivation}

Drawing edges as octilinear paths plays a central role in the design of metro-maps (see e.g., [91819]), which dates back to the 1930's when Henry Beck, an engineering draftsman, designed the first schematic map of London Underground using mostly horizontal, vertical and diagonal segments. Laying out networks in such a way is called octilinear graph drawing, i.e., an octilinear drawing of a (planar) graph $G=(V, E)$ of maximum degree eight is a (planar) drawing $\Gamma(G)$ of $G$ in which each vertex occupies a point on the integer grid and each edge is drawn as a sequence of horizontal, vertical and diagonal line-segments.

For drawings of (planar) graphs to be readable, special care is needed to keep the number of bends small. However, the problem of determining whether a given embedded 8-planar graph (that is, a planar graph of maximum degree eight with given combinatorial embedding) admits a bendless octilinear drawing is NP-hard [17]. This negative result motivated us to study octilinear drawings of low edge complexity, that is, with few bends per edge. Surprisingly enough, very few results relevant to this problem were known, even if the octilinear model has been well-studied in the context of metromap visualization and map schematization (see e.g. [21]). As an immediate byproduct

\footnotetext{
* The work of M.A. Bekos is implemented within the framework of the Action "Supporting Postdoctoral Researchers" of the Operational Program "Education and Lifelong Learning" (Action's Beneficiary: General Secretariat for Research and Technology), and is co-financed by the European Social Fund (ESF) and the Greek State.
}

C. Duncan and A. Symvonis (Eds.): GD 2014, LNCS 8871, pp. 331-342 2014.

(c) Springer-Verlag Berlin Heidelberg 2014 
of a result of Keszegh et al. [13], it turns out that every $d$-planar graph, with $3 \leq d \leq 8$, admits a planar octilinear drawing with at most two bends per edge; see Section 1 On the other hand, every 3-planar graph on five or more vertices admits a planar octilinear drawing in which all edges are bendless [612].

In this paper, we bridge the gap between the two aforementioned results. We prove that every 4-planar graph admits a planar octilinear drawing with at most one bend per edge in cubic area. We also show that every 5-planar graph also admits a planar octilinear drawing with at most one bend per edge, but our construction may require superpolynomial area. Finally, we demonstrate an infinite class of 6-planar graphs whose planar octilinear drawings require at least two bends per edge.

Related Work. The research on the (planar) slope number of graphs focuses on minimizing the number of used slopes (see e.g., [10|13 14 15 16$]$ ). Octilinear drawings can be seen as a special case thereof, since only four slopes are used. Keszegh et al. [13] showed that any $d$-planar graph admits a planar drawing with one bend per edge, in which all edge-segments have at most $2 d$ different slopes. So, for $d=4$ and $d=5$, we reduce the number of different slopes from 8 and 10 to 4 . They also proved that $d$-planar graphs, $d \geq 3$, admit planar drawings with two bends per edge that require at most $\left\lceil\frac{d}{2}\right\rceil$ different slopes. One can transfer this technique to the octilinear model and show that any $d$-planar graph, with $3 \leq d \leq 8$, admits a planar octilinear drawing with two bends per edge. For $d=3$, Di Giacomo et al. [6] recently proved that any 3-planar graph with $n \geq 5$ vertices has a bendless planar drawing with at most 4 different slopes and angular resolution $\pi / 4$ (see also [12]); their approach also yields octilinear drawings.

Tamassia [20] showed that one can minimize the total number of bends in orthogonal drawings of embedded 4-planar graphs. However, minimizing the number of bends over all embeddings of a 4-planar graph is NP-hard [7]. The core of Tamassia's approach is a min-cost flow algorithm that specifies the angles and the bends of the drawing, producing an orthogonal representation, and then computes the actual drawing by specifying the drawing's exact coordinates. Tamassia's algorithm can be employed to produce a bend-minimum octilinear representation for any given embedded 8-planar graph. However, a bend-minimum octilinear representation may not be realizable by a corresponding planar octilinear drawing [4].

Biedl and Kant [2] showed that any 4-planar graph except the octahedron admits a planar orthogonal drawing with at most two bends per edge on an $O\left(n^{2}\right)$ integer grid. Hence, the octilinear drawing model allows us to reduce the number of bends per edge at the cost of an increased area. On the other hand, not all 4-planar graphs admit orthogonal drawings with one bend per edge; however, testing whether a 4-planar graph admits such a drawing can be done in polynomial time [3]. In the context of metromap visualization, several approaches have been proposed to produce metro-maps using octilinear or nearly-octilinear polylines; see e.g., [9]18|19].

Preliminaries. In our algorithms, we incrementally construct the drawings similar to the method of Kant [11]. We first employ the canonical order to cope with triconnected graphs. Then, we extend them to biconnected graphs using the SPQR-tree and to simply connected graphs using the BC-tree. In this section we briefly recall them. 
Definition 1 (Canonical order [11]). For a given triconnected plane graph $G=(V, E)$ let $\Pi=\left(P_{0}, \ldots, P_{m}\right)$ be a partition of $V$ into paths such that $P_{0}=\left\{v_{1}, v_{2}\right\}, P_{m}=$ $\left\{v_{n}\right\}$ and $v_{2} \rightarrow v_{1} \rightarrow v_{n}$ is a path on the outer face of $G$. For $k=0, \ldots, m$ let $G_{k}$ be the subgraph induced by $\cup_{i=0}^{k} P_{i}$ and assume it inherits its embedding from $G$. Partition $\Pi$ is a canonical order of $G$ if for each $k=1, \ldots, m-1$ the following hold: (i) $G_{k}$ is biconnected, (ii) all neighbors of $P_{k}$ in $G_{k-1}$ are on the outer face, of $G_{k-1}$ (iii) all vertices of $P_{k}$ have at least one neighbor in $P_{j}$ for some $j>k . P_{k}$ is called a singleton if $\left|P_{k}\right|=1$ and a chain otherwise.

Definition 2 (BC-tree). The BC-tree $\mathcal{B}$ of a connected graph $G$ has a B-node for each biconnected component of $G$ and a $C$-node for each cutvertex of $G$. Each B-node is connected with the $C$-nodes that are part of its biconnected component.

An SPQR-tree [85] provides information about the decomposition of a biconnected graph into its triconnected components. Every triconnected component is associated with a node $\mu$ in the SPQR-tree $\mathcal{T}$. The triconnected component itself is referred to as the skeleton of $\mu$, denoted by $G_{\mu}^{\text {skel }}=\left(V_{\mu}^{\text {skel }}, E_{\mu}^{\text {skel }}\right)$. We refer to the degree of a vertex $v \in V_{\mu}^{s k e l}$ in $G_{\mu}^{s k e l}$ as $\operatorname{deg}_{\mu}^{\text {skel }}(v)$. We say that $\mu$ is an $R$-node, if $G_{\mu}^{\text {skel }}$ is a simple triconnected graph. A bundle of at least three parallel edges classifies $\mu$ as a $P$-node, while a simple cycle of length at least three classifies $\mu$ as an $S$-node. By construction R-nodes are the only nodes of the same type that are allowed to be adjacent in $\mathcal{T}$. The leaves of $\mathcal{T}$ are formed by the $Q$-nodes. Their skeleton consists of two parallel edges; one of them corresponds to an edge of $G$ and is referred to as real edge. The skeleton edges that are not real are referred to as virtual edges. A virtual edge $e$ in $G_{\mu}^{\text {skel }}$ corresponds to a tree node $\mu^{\prime}$ that is adjacent to $\mu$ in $\mathcal{T}$, more exactly, to another virtual edge $e^{\prime}$ in $G_{\mu^{\prime}}^{s k e l}$. We assume that $\mathcal{T}$ is rooted at a Q-node. Hence, every skeleton (except the one of the root) contains exactly one virtual edge $e=(s, t)$ that has a counterpart in the skeleton of the parent node. We call this edge the reference edge of $\mu$ denoted by $r e f(\mu)$. Its endpoints, $s$ and $t$, are named the poles of $\mu$ denoted by $\mathcal{P}_{\mu}=\{s, t\}$. Every subtree rooted at a node $\mu$ of $\mathcal{T}$ induces a subgraph of $G$ called the pertinent graph of $\mu$ that we denote by $G_{\mu}^{\text {pert }}=\left(V_{\mu}^{\text {pert }}, E_{\mu}^{\text {pert }}\right)$. We abbreviate the degree of a node $v$ in $G_{\mu}^{\text {pert }}$ with $\operatorname{deg}_{\mu}^{\text {pert }}(v)$. The pertinent graph is the subgraph of $G$ for which the subtree describes the decomposition. The following lemmata provide useful properties of SPQR-trees. Due to lack of space, their proofs are given in [1].

Lemma 1. Let $\mu$ be a tree node that is not the root in the SPQR-tree $\mathcal{T}$ of a simple, biconnected, $k$-planar graph $G$ and $\mu^{\prime}$ its parent in $\mathcal{T}$. For $v \in \mathcal{P}_{\mu}$, it holds that $\operatorname{deg}_{\mu}^{\text {pert }}(v) \leq k-2$, if $\mu^{\prime}$ is a $P$ - or an $R$-node and deg $g_{\mu}^{\text {pert }}(v) \leq k-1$ otherwise, i.e. $\mu^{\prime}$ is an $S$ - or a $Q$-node.

Lemma 2. In the SPQR-tree $\mathcal{T}$ of a planar biconnected graph $G=(V, E)$ with $\operatorname{deg}(v)$ $\geq 3$ for every $v \in V$, there exists at least one $Q$-node that is adjacent to a $P$-or an $R$-node.

\section{Octilinear Drawings of 4-Planar Graphs}

In this section, we focus on octilinear drawings of 4-planar graphs. We first consider the triconnected case and then we extend it to biconnected and simply connected graphs. 
The Triconnected Case. Let $G=(V, E)$ be a triconnected 4-planar graph and $\Pi=$ $\left\{P_{0}, \ldots, P_{m}\right\}$ be a canonical order of $G$. We momentarily neglect the edge $\left(v_{1}, v_{2}\right)$ of the first partition $P_{0}$ of $\Pi$ and we start by placing the second partition, say a chain $P_{1}=\left\{v_{3}, \ldots, v_{\left|P_{1}\right|+2}\right\}$, on a horizontal line from left to right. Since $v_{3}$ and $v_{\left|P_{1}\right|+2}$ are adjacent to $v_{1}$ and $v_{2}$, we place $v_{1}$ to the left of $v_{3}$ and $v_{2}$ to the right of $v_{\left|P_{1}\right|+2}$. So, they form a single chain where all edges are drawn using horizontal line-segments that are attached to the east and west port at their endpoints. The case where $P_{1}$ is a singleton is analogous. Having laid out the base of our drawing, we now place in an incremental manner the remaining partitions. Assume that we have already constructed a drawing for $G_{k-1}$ and we now have to place $P_{k}$, for some $k=2, \ldots, m-1$.

In case where $P_{k}=\left\{v_{i}, \ldots, v_{j}\right\}$ is a chain of $j-i+1$ vertices, we draw them from left to right along a horizontal line one unit above $G_{k-1}$. Since $v_{i}$ and $v_{j}$ are the only vertices that are adjacent to vertices in $G_{k-1}$, both only to one, we place the chain between those two as in Fig 1 a The port used at the endpoints of $P_{k}$ in $G_{k-1}$ depends on the following rule: Let $v_{i}^{\prime}\left(v_{j}^{\prime}\right.$, resp.) be the neighbor of $v_{i}\left(v_{j}\right.$, resp.) in $G_{k-1}$. If the edge $\left(v_{i}, v_{i}^{\prime}\right)\left(\left(v_{j}, v_{j}^{\prime}\right)\right.$, resp.) is the last to be attached to vertex $v_{i}^{\prime}\left(v_{j}^{\prime}\right.$, resp.), i.e., there is no vertex $v$ in $P_{l} \in \Pi, l>k$ such that $\left(v_{i}^{\prime}, v\right) \in E\left(\left(v_{j}^{\prime}, v\right) \in E\right.$, resp.), then we use the northern port of $v_{i}^{\prime}\left(v_{j}^{\prime}\right.$, resp.). Otherwise, we choose the north-east port for $\left(v_{i}, v_{i}^{\prime}\right)$ or the north-west port for $\left(v_{j}, v_{j}^{\prime}\right)$.

In case of a singleton $P_{k}=\left\{v_{i}\right\}$, we can apply the previous rule if the singleton is of degree three. However, if $v_{i}$ is of degree four, then we may have to deal with an additional third edge $\left(v_{i}, v\right)$ that connects $v_{i}$ with $G_{k-1}$. However, we may assume that $v$ lies between the other two endpoints, thus, we place $v_{i}$ such that $x\left(v_{i}\right)=x(v)$. This enables us to draw $\left(v_{i}, v\right)$ as a vertical line-segment; see Fig $1 \mathrm{~b}$.

The above procedure is able to handle all chains and singletons except the last partition $P_{m}$, as $v_{n}$ may have 4 edges pointing downwards. We exclude $\left(v_{n}, v_{1}\right)$ and draw $v_{n}$ as an ordinary singleton. Then, we shift $v_{1}$ to the left and up as in Fig $1 \mathrm{c}$ and draw $\left(v_{1}, v_{n}\right)$ as a horizontal-vertical segment combination. Vertex $v_{2}$ is analogously moved. The drawings of the remaining edges incident to $v_{n}$ are depicted in Fig $1 \mathrm{C}$

A cut is a $y$-monotone continuous curve that crosses only horizontal segments and divides the current drawing into a left and a right part. Since every edge, except the ones drawn as vertical line-segments, contains exactly one horizontal segment, we can shift the right part of the drawing that is defined by the cut further to the right while keeping the left part of the drawing on place and the result remains a valid octilinear drawing.

To compute the $\mathrm{x}$-coordinates, we first assign consecutive $\mathrm{x}$-coordinates to the first two partitions. We may have to stretch the drawing in two cases: (i) when we introduce a chain, say $P_{k}$, as it may not fit into the gap defined by its two adjacent vertices in $G_{k-1}$, and, (ii) when an edge that contains a diagonal segment is to be drawn, to prevent it from intersecting any horizontal-vertical combinations in the face below it. We can cope with both cases by horizontally stretching the drawing by a factor that is bounded by the current height of the drawing. Since the height of the resulting drawing is bounded by $|\Pi|=O(n)$, it follows that in the worst case its width is $O\left(n^{2}\right)$. Note that our algorithm produces drawings that have a linear number of bends in total (in particular, exactly $2|\Pi|=O(n)$ bends). One can prove that this bound is asymptotically tight (see [1]). We are now ready to state the main theorem of this subsection. 


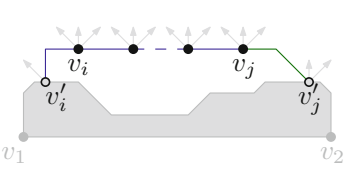

(a)

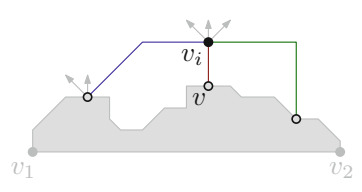

(b)

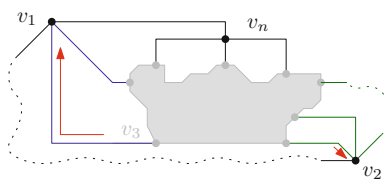

(c)

Fig. 1. (a) Horizontal placement of a chain $P_{k}=\left\{v_{i}, \ldots, v_{j}\right\}$. (b) Placement of a singleton $P_{k}=\left\{v_{i}\right\}$ with degree four. (c) Final layout after repositioning $v_{1}$ and $v_{2}$ (the shape of the dotted edges can be obtained by extending the stubs until they intersect).

Theorem 1. Given a triconnected 4-planar graph $G$, we can compute in $O(n)$ time an octilinear drawing of $G$ with at most 1 bend per edge on an $O\left(n^{2}\right) \times O(n)$ integer grid.

Proof. In order to keep the time complexity of our algorithm linear, we employ a simple trick. We assume that any two adjacent points of the underlying integer grid are by $n$ units apart in the horizontal direction and by one unit in the vertical direction. This a priori ensures that all edges that contain a diagonal segment will not be involved in crossings and simultaneously does not affect the total area of the drawing, which asymptotically remains cubic. On the other hand, the advantage of this approach is that we can use the shifting method of Kant [11] to cope with the introduction of chains in the drawing, that needs $O(n)$ time in total by keeping relative coordinates that can be efficiently updated and computing the absolute values only at the last step.

The Biconnected Case. Consider a node $\mu$ in the rooted SPQR-tree $\mathcal{T}$ of $G$ with poles $\mathcal{P}_{\mu}=\{s, t\}$. In the drawing of $G_{\mu}^{p e r t}, s$ should be located at the upper-left and $t$ at the lower-right corner of the drawing's bounding box with a port assignment as in Fig 2a. We also assume that the edges incident to $s$ ( $t$, resp.) use the western (eastern, resp.) port at their other endpoint, except of the northern (southern, resp.) most edge which may use the north (south, resp.) port instead. In that case we refer to $s$ and $t$ as fixed; see $\bar{e}_{s}, \bar{e}_{t}$ in Fig $2 \mathrm{a}$. We maintain the following invariants:

IP-1: The width (height) of the drawing of $\mu$ is quadratic (linear) in the size of $G_{\mu}^{\text {pert }} . s$ is located at the upper-left; $t$ at the lower-right corner of the drawing's bounding box.

IP-2: If $\operatorname{deg}_{\mu}^{\text {pert }}(s) \geq 2, s$ is fixed; $t$ is fixed if $d e g_{\mu}^{\text {pert }}(t)=3$ and $\mu$ 's parent is not the root.

IP-3: The edges that are incident at $s$ and $t$ in $G_{\mu}^{\text {pert }}$ use the south, south-east and east ports at $s$ and the north, north-west and west port at $t$, resp. If $s$ or $t$ is not fixed, incident edges are attached at their other endpoints via the west and east port, respectively. If $s$ or $t$ is fixed, the northern-most edge at $s$ and the southern-most edge at $t$ may use the north (south, resp.) port at its other endpoint.

The port assignment, i.e. IP- 3 , guarantees the ability to stretch the drawing horizontally even in the case where both poles are fixed. Furthermore, IP-2 is interchangeable in the following sense: If $\operatorname{deg}_{\mu}^{\text {pert }}(s)=2$ and $\operatorname{deg}_{\mu}^{\text {pert }}(t)=1$, then $s$ is fixed but $t$ is 


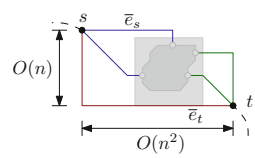

(a)

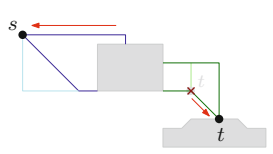

(b)

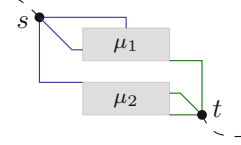

(c)

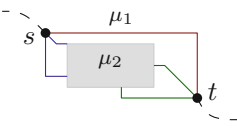

(d)

Fig. 2. (a) Schematic view of the layout requirements. (b) Creating a nose at $t$. (c) First P-node subcase without an $(s, t)$-edge but $s$ might be fixed in a child $\mu_{1}$. (d) Second P-node subcase with an $(s, t)$-edge where $t$ might get fixed in a child $\mu_{2}$.

not. But, if we relabel $s$ and $t$ such that $t^{\prime}=s$ and $s^{\prime}=t$, then $\operatorname{deg}_{\mu}^{\text {pert }}\left(s^{\prime}\right)=1$ and $\operatorname{deg}_{\mu}^{\text {pert }}\left(t^{\prime}\right)=2$. By IP 2 , we can create a drawing where both $s^{\prime}$ and $t^{\prime}$ are not fixed and located in the upper-left and lower-right corner of the drawing's bounding box. Afterwards, we mirror the resulting layout vertically and horizontally to obtain one where $s$ and $t$ are in their respective corners and not fixed. For a non-fixed vertex, we introduce an operation referred to as forming or creating a nose; see Fig $2 \mathrm{~b}$, where $t$ has been moved downwards at the cost of a bend. As a result, its west port is no longer occupied.

First consider the case where $\mu$ is a P-node. If there is no $(s, t)$-edge, then we draw the children of $\mu$ from top to bottom such that a possible child in which $s$ is fixed, is drawn topmost (see $\mu_{1}$ in Fig $2 \mathrm{c}$ ). If there is an $(s, t)$-edge, then we draw it at the top and afterwards the remaining children of $\mu$ (see Fig 2d). This is possible only if $s$ is not fixed in any of the other children. Let $\mu^{\prime}$ be such a potential child where $s$ is fixed, i.e., $\operatorname{deg}_{\mu^{\prime}}^{\text {pert }}(s)=2$, and thus, the only child that remains to be drawn. Here, we use the property of interchangeability to "unfix" $s$ in $\mu^{\prime}$. As a result $s$ can form a nose, whereas $t$ may now be fixed in $\mu^{\prime}$ when ${ }^{\prime} e g_{\mu^{\prime}}^{\text {pert }}(t)=2$ holds, as in Fig 2d. However, then $\operatorname{deg}_{\mu}^{\text {pert }}(t)=3$ follows. Notice that the presence of an $(s, t)$-edge implies that the parent of $\mu$ is not the root of $\mathcal{T}$, since this would induce a pair of parallel edges. Hence, by IP -2 we are allowed to fix $t$ in $\mu$. Port assignment and area requirements comply in both cases with our invariant properties.

In the case where $\mu$ is an S-node, we place the drawings of its children, say $\mu_{1}, \ldots, \mu_{\ell}$ in a "diagonal manner" such that their corners touch as in Fig $3 \mathrm{a}$ In case of Q-nodes being involved, we draw their edges as horizontal segments (see, e.g., $\left(v_{3}, v_{4}\right)$ in Fig 3a). $s$ and $t$ inherit their port assignment and pertinent degree from $\mu_{1}$ and $\mu_{\ell}$, respectively. So, $s$ ( $t$, resp.) is fixed in $\mu$, if it is fixed in $\mu_{1}$ ( $\mu_{\ell}$, resp.). By IP-2 $t$ is not allowed to be fixed in the case where the parent of $\mu$ is the root of $\mathcal{T}$. However, from Lemma 2 we can choose the root such that $t$ is not fixed in that case, and thus, complies with IP-2. Since we only concatenated the drawings of the children, IP-1 and IP- 3 are satisfied.

For the case where $\mu$ is an R-node with poles $\mathcal{P}_{\mu}=\{s, t\}$, we follow the idea of the triconnected algorithm and describe only the required modifications. We assume the worst case where no child of $\mu$ is a Q-node. Let $\mu_{u v}$ be the child that is represented by the virtual edge $(u, v) \in E_{\mu}^{\text {skel }}$. Due to Lemma1, $\operatorname{deg}_{\mu_{u v}}^{\text {pert }}(u) \leq 2$ and $\operatorname{deg}_{\mu_{u v}}^{\text {pert }}(v) \leq 2$ holds. By IP-2 we may assume that either $u$ or $v$ is fixed in $\mu_{u v}$ and choose the first partition in the canonical ordering to be $P_{0}=\{s, t\}$.

In case of a chain, say $P_{k}=\left\{v_{i}, \ldots, v_{j}\right\}$ with two neighbors $v_{i}^{\prime}$ and $v_{j}^{\prime}$ in $G_{k-1}$, we have to replace two types of edges with the drawings of the corresponding children: 


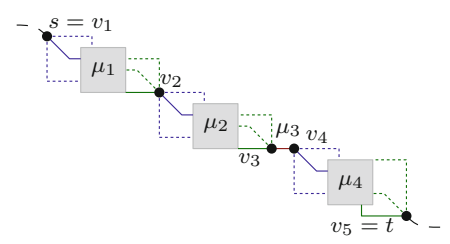

(a)

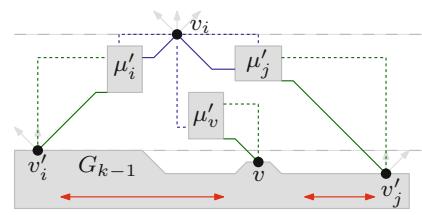

(c)

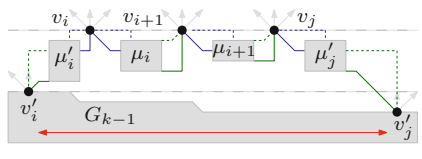

(b)

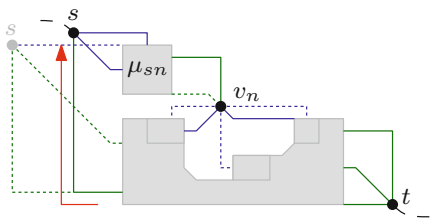

(d)

Fig. 3. (a) S-node with children $\mu_{1}, \ldots, \mu_{4} ; \mu_{3}$ is a Q-node representing the edge $\left(v_{3}, v_{4}\right)$. Optional edges are drawn dotted. (b) Example for a chain $v_{i}, \ldots, v_{j}$ with virtual edges representing $\mu_{i}, \ldots, \mu_{j-1}$ in the R-node case. (c) Singleton $v_{i}$ with possibly three incident virtual edges representing $\mu_{i}^{\prime}, \mu_{v}^{\prime}, \mu_{j}^{\prime}$. (d) Placing $v_{n}$ and moving up $s$ which might be fixed in $\mu_{s n}$.

the edges $\left(v_{i}, v_{i+1}\right), \ldots,\left(v_{j-1}, v_{j}\right)$ representing the children $\mu_{i}, \ldots, \mu_{j-1}$ and $\left(v_{i}^{\prime}, v_{i}\right)$ $\left(\left(v_{j}, v_{j}^{\prime}\right)\right.$ resp.) representing $\mu_{i}^{\prime}\left(\mu_{j}^{\prime}\right.$ resp.). We place the vertices of $P_{k}$ on a horizontal line high enough above $G_{k-1}$ so that every drawing fits in-between. Then, we insert the drawings aligned below the horizontal line and choose for $i \leq l<j, v_{l}$ to be the fixed node in $\mu_{l}$, whereas in $\mu_{i}^{\prime}$ ( $\mu_{j}^{\prime}$ resp.), we set $v_{i}$ ( $v_{j}$ resp.) to be fixed. So, for $i \leq l<j$, $v_{l+1}$ may form a nose in $\mu_{l}$ pointing upwards while $v_{i}^{\prime}$ and $v_{j}^{\prime}$ form each one downwards as in Fig $3 \mathrm{~b}$. For the extra height and width, we stretch the drawing horizontally.

In case where $P_{k}=\left\{v_{i}\right\}, i \neq n$ is a singleton, we only outline the difference which is a possible third edge $\left(v_{i}, v\right)$ to $G_{k-1}$ representing say $\mu_{v}^{\prime}$. While the other two involved children, say $\mu_{i}^{\prime}$ and $\mu_{j}^{\prime}$, are handled as in the chain-case, $\mu_{v}^{\prime}$ requires extra height. We place $v_{i}$ so that $\mu_{v}^{\prime}$ fits below $\mu_{j}^{\prime}$ as in Fig $3 \mathrm{c}$. Notice that $\operatorname{deg}_{\mu_{v}^{\prime}}^{\text {pert }}\left(v_{i}\right)=1$ and by IP-2 both $v_{i}$ and $v$ are not fixed in $\mu_{v}^{\prime}$. So, forming a nose at $v_{i}$ and $v$ is feasible.

For the last singleton $P_{k}=\left\{v_{n}\right\}$, observe that since $s, t \in P_{0}$, both have not been fixed. As in the triconnected algorithm we move $s=v_{1}$ above $v_{n}$ as in Fig $3 \mathrm{~d}$ to accommodate the drawing of the child $\mu_{s n}$ represented by the edge $\left(s, v_{n}\right)$. Since we may require $v_{n}$ to form a nose in $\mu_{s n}$ as in Fig $3 \mathrm{~d}$. we choose $s$ to be fixed in $\mu_{s n}$. By IP-2 we are allowed to fix $s$ since $t$ remains unfixed. Although some diagonal segments may force us to stretch the whole drawing by its height, the height of the drawing has been kept linear in the size of $G_{\mu}^{\text {pert }}$. Since we increase the width by the height a constant number of times per step, the resulting width remains quadratic.

If there is a vertex $v \in V$ with $\operatorname{deg}(v) \leq 3$, then we root $\mathcal{T}$ at a Q-node $\mu$ that represents one of its three incident edges and orient the poles $\{s, t\}$ such that $t=v$. So, for the child $\mu^{\prime}$ of $\mu$ follows $\operatorname{deg}_{\mu^{\prime}}^{\text {pert }}(t) \leq 2$. If $\operatorname{deg}(v)=4$ for every $v \in V$, then we root $\mathcal{T}$ at a Q-node that is not adjacent to an S-node, which exists due to Lemma 2 In both cases, we may form a nose with $t$ pointing downwards. 
Theorem 2. Given a biconnected 4-planar graph $G$, we can compute in $O(n)$ time an octilinear drawing of $G$ with at most 1 bend per edge on an $O\left(n^{2}\right) \times O(n)$ integer grid.

Proof. The SPQR-tree $\mathcal{T}$ can be computed in $O(n)$-time and its size is linear to the size of $G[8]$. The pertinent degrees of the poles at every node can be pre-computed by a bottom-up traversal of $\mathcal{T}$. Drawing a P-node requires constant time; $\mathrm{S}$ - and R-nodes require time linear to the size of the skeleton. However, the sum over all skeleton edges is linear, as every virtual edge corresponds to a tree node.

The Simply Connected Case. The main idea of our algorithm is to root the BC-tree at some arbitrary B-node. With exception of the root, every B-node contains a designated cut vertex that links it to the parent. Similar to the biconnected case, we define an invariant for the drawing of a subtree. The cut vertex that links the subtree to the parent is located in the upper left corner of the bounding box. Due to lack of space, we only state the main result; its proof is given in [1].

Theorem 3. Given a connected 4-planar graph $G$, we can compute in $O(n)$ time an octilinear drawing of $G$ with at most 1 bend per edge on an $O\left(n^{2}\right) \times O(n)$ integer grid.

\section{Octilinear Drawings of 5-Planar Graphs}

In this section, we focus on planar octilinear drawings of 5-planar graphs. As in Section 2 we first consider the case of triconnected 5-planar graphs and then we extend our approach first to biconnected and then to the simply connected graphs.

The Triconnected Case. Let $G=(V, E)$ be a triconnected 5-planar graph and $\Pi=$ $\left\{P_{0}, \ldots, P_{m}\right\}$ be a canonical order of $G$. We place partitions $P_{0}$ and $P_{1}$ similar to the 4-planar case. Assume that we have already constructed a drawing for $G_{k-1}$ which is stretchable in the following sense: If $e \in E\left(G_{k-1}\right)$ is an edge incident to the outer face, then there is a cut which crosses $e$ and can be utilized to horizontally stretch the drawing of $G_{k-1}$. In other words, one can define a cut through every edge incident to the outer face of $G_{k-1}$ (stretchability-invariant).

If $P_{k}=\left\{v_{i}, \ldots, v_{j}\right\}$ is a chain, it is placed exactly as in the case of 4-planar graphs, but with different port assignment. Among the northern available ports of vertex $v_{i}^{\prime}$ $\left(v_{j}^{\prime}\right.$, resp.), edge $\left(v_{i}, v_{i}^{\prime}\right)\left(\left(v_{j}, v_{j}^{\prime}\right)\right.$, resp.) uses the eastern-most unoccupied port of $v_{i}^{\prime}$ (western-most unoccupied port of $v_{j}^{\prime}$, resp.); see Fig 4a, If $P_{k}$ does not fit into the gap between $v_{i}^{\prime}$ and $v_{j}^{\prime}$ in $G_{k-1}$, then we horizontally stretch $G_{k-1}$ between $v_{i}^{\prime}$ and $v_{j}^{\prime}$ to ensure that the horizontal distance between $v_{i}^{\prime}$ and $v_{j}^{\prime}$ is at least $\left|P_{k}\right|+1$. This can be done due to the stretchability-invariant, as both $v_{i}^{\prime}$ and $v_{j}^{\prime}$ are on the outer face of $G_{k-1}$. Potential crossings introduced by edges of $P_{k}$ containing diagonal segments can be eliminated by employing similar cuts to the ones presented in the 4-planar case. So, we may assume that $G_{k}$ is plane. Also, $G_{k}$ complies with the stretchability-invariant, as one can define a cut that crosses any of the newly inserted edges of $P_{k}$ and then follows one of the cuts of $G_{k-1}$ that crosses an edge between $v_{i}^{\prime}$ and $v_{j}^{\prime}$. 


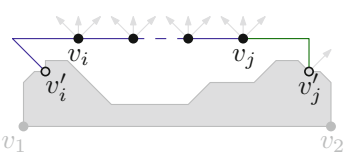

(a)

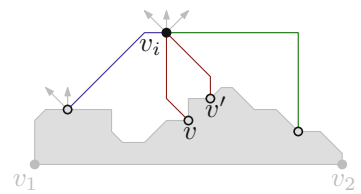

(b)

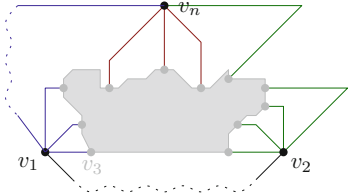

(c)

Fig. 4. (a) Horizontal placement of a chain $P_{k}=\left\{v_{i}, \ldots, v_{j}\right\}$. (b) Placement of a singleton $P_{k}=\left\{v_{i}\right\}$ of degree five. (c) Final layout (the shape of the dotted edges can be obtained by extending the stubs until they intersect).

In case of a singleton $P_{k}=\left\{v_{i}\right\}$ of degree 5 , we have to deal with two additional edges (called nested) that connect $v_{i}$ with $G_{k-1}$, say $\left(v_{i}, v\right)$ and $\left(v_{i}, v^{\prime}\right)$; see Fig $4 \mathrm{~b}$, Such a pair of edges does not always allow vertex $v_{i}$ to be placed along the next available horizontal grid line. A careful case analysis on the type of ports that are unoccupied at $v$ and $v^{\prime}$ in conjunction with the fact that $G_{k-1}$ is horizontally stretchable shows that we can find a feasible placement for $v_{i}$. Potential crossings due to the remaining edges incident to $v_{i}$ are eliminated by employing similar cuts to the ones presented in the 4planar case. So, $G_{k}$ is planar. Also, $G_{k}$ complies with the stretchability-invariant. The last partition $P_{m}=\left\{v_{n}\right\}$ is treated in the same way, even if $v_{n}$ can be incident to three nested edges. Since $v_{1}$ and $v_{2}$ are along a common horizontal line, $\left(v_{1}, v_{2}\right)$ can be drawn using two diagonal segments that form a bend pointing downwards; see Fig $4 \mathrm{c}$. Note that our algorithm may result in drawings of super-polynomial area, as proven in [1].

Theorem 4. Given a triconnected 5-planar graph $G$, we can compute in $O\left(n^{2}\right)$ time an octilinear drawing of $G$ with at most one bend per edge.

Proof. We can no longer use the shifting method of Kant [11], since the $x$ - and $y$ coordinates are not independent. However, the computation of each cut can be done in linear time, which implies that our drawing algorithm needs $O\left(n^{2}\right)$ time in total.

The Biconnected Case. For the 4-planar case we defined several invariants in order to keep the area of the resulting drawings polynomial. Since we drop this requirement now we can define a simpler new invariant for the biconnected 5-planar case. When considering a node $\mu$ in $\mathcal{T}$ and its poles $\mathcal{P}_{\mu}=\{s, t\}$, then in the drawing of $G_{\mu}^{\text {pert }}, s$ and $t$ are horizontally aligned at the bottom of the drawing's bounding box as in Fig 5a. If an $(s, t)$-edge is present, it can be drawn at the bottom. An $(s, t)$-edge only occurs in the pertinent graph of a P-node (and Q-node). We use the term fixed for a pole-node that is not allowed to form a nose. We maintain the following properties through the recursive construction process: In S- and R- nodes, $s$ and $t$ are not fixed. In P- and Q-nodes, only one of them is fixed, say $s$. But as in the 4-planar case, we may swap their roles.

If $\mu$ is a P-node, then it has at most 4 children; one of them might be a Q-node, i.e., an $(s, t)$-edge, which can be drawn at the bottom as a horizontal segment. Since P-nodes are not adjacent to each other in $\mathcal{T}$, the remaining children are S- or R-nodes. By our invariant we may form noses enabling us to stack them as in Fig 5 b. 


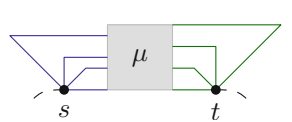

(a)

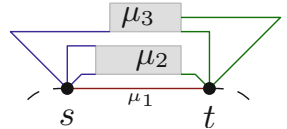

(b)

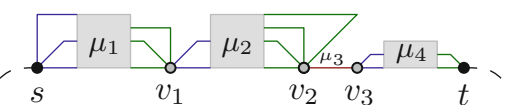

(c)

Fig. 5. (a) Layout specification; $s$ and $t$ are located at the bottom. (b) P-node with an $(s, t)$ edge from a Q-node $\mu_{1} . s$ and $t$ form a nose in $\mu_{2}, \mu_{3}$. (c) S-node example with four children $\mu_{1}, \ldots, \mu_{4}$.

In case where $\mu$ is an $\mathrm{S}$-node, we align its children $\mu_{1}, \ldots, \mu_{l}$ horizontally; see Fig $5 \mathrm{c}$. The poles inherit their pertinent degree from the children. The same holds for the property of being fixed. However, by our new invariant this is forbidden, as it requires that $s$ and $t$ are not fixed. It is easy to see that when $\mu_{1}$ is a P-node, $s$ is fixed by the invariant in $\mu_{1}$. In this case, we swap the roles of the poles in $\mu_{1}$ such that $s$ is not fixed. However, the other pole of $\mu_{1}$, say $v_{1}$, is fixed now. Since the skeleton of an $\mathrm{S}$-node is a cycle of length at least three, $v_{1} \neq t$. So, $s$ and $t$ are not fixed in the resulting drawing.

To compute a layout of an R-node $\mu$, we employ the triconnected algorithm (with $s=v_{1}$ and $\left.t=v_{2}\right)$. Let $\mu_{e}$ be a child of $\mu$ that corresponds to virtual edge $e=$ $(u, v)$ in $G_{\mu}^{\text {skel }}$. Then, $\operatorname{deg}_{\mu_{e}}^{\text {pert }}(u), \operatorname{deg}_{\mu_{e}}^{\text {pert }}(v) \leq 3$. When inserting the drawing of $G_{\mu_{e}}^{\text {pert }}$, we require at most three consecutive ports at $u$ and $v$ for the additional edges. As the triconnected algorithm assigns ports in a consecutive manner based on the relative position of the endpoints, we modify the port assignment so that an edge may have more than one port assigned. To do so, we assign each edge $e=(u, v)$ in $G_{\mu}^{\text {skel }}$ a pair $\left(\operatorname{deg}_{\mu_{e}}^{\text {pert }}(u), \operatorname{deg}_{\mu_{e}}^{\text {pert }}(v)\right) \in\{1,2,3\}^{2}$ that reflects the number of ports required by this edge at its endpoints. Then, we extend the triconnected algorithm such that when a port of $u$ is assigned to an edge $e=(u, v)$, $\operatorname{deg}_{\mu_{e}}^{\text {pert }}(u)-1$ additional consecutive ports in clockwise or counterclockwise order are reserved. The direction depends on the different types of edges that we will discuss next.

The simplest type of edges are the ones among consecutive vertices $v_{i}, v_{i+1}$ of a chain. For each such edge we reserve the additional ports at $v_{i}$ in counterclockwise and at $v_{i+1}$ in clockwise order; see Fig 6a. So, we can later plug the drawing of the children into the layout as in Fig $6 \mathrm{~b}$ without forming noses. In the same manner, we reserve the ports for the edges that connect $P_{k}=\left\{v_{i}, \ldots, v_{j}\right\}$ to $v_{i}^{\prime}$ and $v_{j}^{\prime}$ in $G_{k-1}$ (where $P_{k}$ is singleton or chain), i.e., at $v_{i}$ clockwise, $\left(v_{j}\right.$ counter-clockwise, resp.) and at $v_{i}^{\prime}$ counter-clockwise ( $v_{j}^{\prime}$ clockwise); see Fig 6c. In case where $\left(v_{i}, v_{i}^{\prime}\right)$ or $\left(v_{j}, v_{j}^{\prime}\right)$ is a virtual edge, we choose the poles such that $v_{i}\left(v_{j}\right.$ resp.) is fixed in $\mu_{\left(v_{i}, v_{i}^{\prime}\right)}\left(\mu_{\left(v_{j}, v_{j}^{\prime}\right)}\right.$ resp.). Thus, we can create a nose with $v_{i}^{\prime}\left(v_{j}^{\prime}\right.$ resp.). Having exactly the ports required at both endpoints, we insert the drawing by replacing the bend with a nose as in Fig 6d. The remaining edges from $P_{k}$ to $G_{k-1}$ in case of a singleton $P_{k}=\left\{v_{i}\right\}$ are handled similarly; see Fig 6 . During the replacement of the edges, the fixed vertex is always the upper one. The only exception are the horizontal drawn edges of a chain, for which it does not matter which one is fixed. Finally, we $\operatorname{root} \mathcal{T}$ at an arbitrarily chosen Q-node representing a real edge $(s, t)$. By our invariant we may construct a drawing with $s$ and $t$ at the bottom of the drawing's bounding box, so that $(s, t)$ has a $90^{\circ}$ bend downwards. 


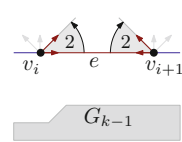

(a)

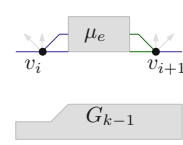

(b)

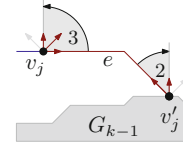

(c)

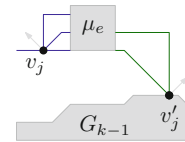

(d)

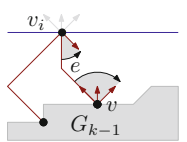

(e)

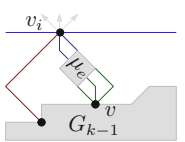

(f)

Fig. 6. (a) Virtual edge $e=\left(v_{i}, v_{i+1}\right)$ connecting two consecutive vertices of a chain. At both endpoints the drawing of $\mu_{e}$ requires two ports. (b) Replacing $e$ in (a) with the corresponding drawing of the child $\mu_{e}$. (c) Example of an edge $e=\left(v_{j}, v_{j}^{\prime}\right)$ that requires three ports at $v_{j}$ and two at $v_{j}^{\prime}$. (d) Inserting the drawing of $\mu_{e}$ into (c) with $v_{j}$ being fixed and $v_{j}^{\prime}$ forming a nose. (e) Reserving ports for the nested edges. A single port for a real edge is reserved and then two ports for the virtual edge $\mathrm{e}=\left(v_{i}, v\right)$. (f) Final layout after inserting the drawing of $\mu_{e}$.

Theorem 5. Given a biconnected 5-planar graph $G$, we can compute in $O\left(n^{2}\right)$ time an octilinear drawing of $G$ with at most one bend per edge.

Proof. The ability to rotate and scale suffices to extend the result from 4-planar to 5planar at the expense of the area. Similar to the 4-planar case, computing $\mathcal{T}$ takes linear time. Hence, the overall runtime is governed by the triconnected algorithm.

The Simply Connected Case. Due to lack of space, we outline the differences in comparison to the 4-planar case in [1]. Here, we simply state the main theorem.

Theorem 6. Given a connected 5-planar graph $G$, we can compute in $O\left(n^{2}\right)$ time an octilinear drawing of $G$ with at most one bend per edge.

\section{A Note on Octilinear Drawings of 6-Planar Graphs}

In this section, we show that it is not always possible to construct a planar octilinear drawing of a given 6-planar graph with at most one bend per edge.

Theorem 7. There exists an infinite class of 6-planar graphs which do not admit planar octilinear drawings with at most one bend per edge.

Sketch of Proof. Due to lack of space the detailed proof of this theorem is given in [1]. The main idea is to construct an infinite class of maximal 6-planar graphs, whose outer face is always delimited by exactly three vertices, say $v, v^{\prime}$ and $v^{\prime \prime}$, such that $\operatorname{deg}(v)=$ $\operatorname{deg}\left(v^{\prime}\right)=6$ and $5 \leq \operatorname{deg}\left(v^{\prime \prime}\right) \leq 6$. Then, it is not difficult to prove that it is not feasible to draw all edges incident to the outer face with at most one bend per edge.

\section{Conclusions}

We presented algorithms for the construction of planar octilinear drawings with at most one bend per edge for 4- and 5-planar graphs. Our work raises several open problems: (i) Is it possible to construct planar octilinear drawings of 4-planar (5-planar) graphs with at most one bend per edge in $o\left(n^{3}\right)$ (polynomial, resp.) area? (ii) Does any trianglefree 6-planar graph admit a planar octilinear drawing with at most one bend per edge? (iii) What is the number of necessary slopes for bendless drawings of 4-planar graphs? 


\section{References}

1. Bekos, M.A., Gronemann, M., Kaufmann, M., Krug, R.: Planar octilinear drawings with one bend per edge. Arxiv report arxiv.org/abs/1408.5920 (2014)

2. Biedl, T.C., Kant, G.: A better heuristic for orthogonal graph drawings. In: van Leeuwen, J. (ed.) ESA 1994. LNCS, vol. 855, pp. 24-35. Springer, Heidelberg (1994)

3. Bläsius, T., Krug, M., Rutter, I., Wagner, D.: Orthogonal graph drawing with flexibility constraints. Algorithmica 68(4), 859-885 (2014)

4. Bodlaender, H.L., Tel, G.: A note on rectilinearity and angular resolution. Journal of Graph Algorithms and Applications 8(1), 89-94 (2004)

5. Di Battista, G., Tamassia, R.: On-line graph algorithms with SPQR-trees. In: Paterson, M. (ed.) ICALP 1990. LNCS, vol. 443, pp. 598-611. Springer, Heidelberg (1990)

6. Di Giacomo, E., Liotta, G., Montecchiani, F.: The planar slope number of subcubic graphs. In: Pardo, A., Viola, A. (eds.) LATIN 2014. LNCS, vol. 8392, pp. 132-143. Springer, Heidelberg (2014)

7. Garg, A., Tamassia, R.: On the computational complexity of upward and rectilinear planarity testing. SIAM Journal on Computing 31(2), 601-625 (2001)

8. Gutwenger, C., Mutzel, P.: A linear time implementation of SPQR-trees. In: Marks, J. (ed.) GD 2000. LNCS, vol. 1984, pp. 77-90. Springer, Heidelberg (2001)

9. Hong, S.H., Merrick, D., do Nascimento, H.A.D.: Automatic visualisation of metro maps. Journal of Visual Languages and Computing 17(3), 203-224 (2006)

10. Jelínek, V., Jelínková, E., Kratochvíl, J., Lidický, B., Tesar, M., Vyskocil, T.: The planar slope number of planar partial 3-trees of bounded degree. Graphs and Combinatorics 29(4), 981-1005 (2013)

11. Kant, G.: Drawing planar graphs using the lmc-ordering. In: 33rd Annual Symposium on Foundations of Computer Science (FOCS 1992), pp. 101-110. IEEE (1992)

12. Kant, G.: Hexagonal grid drawings. In: Mayr, E.W. (ed.) WG 1992. LNCS, vol. 657, pp. 263-276. Springer, Heidelberg (1993)

13. Keszegh, B., Pach, J., Pálvölgyi, D.: Drawing planar graphs of bounded degree with few slopes. SIAM Journal of Discrete Mathematics 27(2), 1171-1183 (2013)

14. Keszegh, B., Pach, J., Pálvölgyi, D., Tóth, G.: Drawing cubic graphs with at most five slopes. Computational Geometry 40(2), 138-147 (2008)

15. Lenhart, W., Liotta, G., Mondal, D., Nishat, R.I.: Planar and plane slope number of partial 2-trees. In: Wismath, S., Wolff, A. (eds.) GD 2013. LNCS, vol. 8242, pp. 412-423. Springer, Heidelberg (2013)

16. Mukkamala, P., Pálvölgyi, D.: Drawing cubic graphs with the four basic slopes. In: van Kreveld, M., Speckmann, B. (eds.) GD 2011. LNCS, vol. 7034, pp. 254-265. Springer, Heidelberg (2011)

17. Nöllenburg, M.: Automated drawings of metro maps. Tech. Rep. 2005-25, Fakultät für Informatik, Universität Karlsruhe (2005)

18. Nöllenburg, M., Wolff, A.: Drawing and labeling high-quality metro maps by mixed-integer programming. IEEE Transactions on Visualization and Computer Graphics 17(5), 626-641 (2011)

19. Stott, J.M., Rodgers, P., Martinez-Ovando, J.C., Walker, S.G.: Automatic metro map layout using multicriteria optimization. IEEE Transactions on Visualization and Computer Graphics 17(1), 101-114 (2011)

20. Tamassia, R.: On embedding a graph in the grid with the minimum number of bends. SIAM Journal of Computing 16(3), 421-444 (1987)

21. Wolff, A.: Graph drawing and cartography. In: Tamassia, R. (ed.) Handbook of Graph Drawing and Visualization, ch. 23, pp. 697-736. CRC Press (2013) 